\title{
Neuromuscular diseases in the pandemic age: 6 months of experience of a newborn clinical center
}

\author{
Francesco Barbato ${ }^{1}\left[\right.$. Giovanni Colacicco ${ }^{1} \cdot$ Giorgia Bruno $^{1}$ - Domenico Ippolito ${ }^{1,2}$. Francesca Siani ${ }^{1}$. \\ Antonio Di Masi ${ }^{1}$. Vincenzo Pota ${ }^{1,3}$
}

Received: 11 April 2021 / Accepted: 9 January 2022 / Published online: 15 February 2022

(c) Fondazione Società Italiana di Neurologia 2022

Chronic neurological diseases represent a growing challenge in terms of health and social repercussions. Fragile patients, as patients affect by chronic neuromuscular disease, are those with high risk of developing severe forms of SARS-COV2 infection. Furthermore, the COVID-19 pandemic has aggravated the complexity of the process of taking care of these patients, despite the ongoing therapeutic advances. The implementation of telemedicine and remote consultation has only partly resolved the reduction in specialist consultation, medical house assistance, and therapeutic adherence $[1,2]$. The aim of Neuromuscular Omnicenter (NeMO) is to diagnose and treat neuromuscular disease, treat the acute complication of that disease as lung failure, aritmia, heart failure and so on and finally give a post-acute rehabilitative assistance. All these objectives are conducted thought a multidisciplinary team (intensivist, neurologist, cardiologist, and physiatrist). For this reason, all the patients who arrived in the ward came directly from home after having carried out a molecular swab to search for COVID-19 in their home, without going through the emergency department.

Although this project has been a reality in Italy for several years, the opening of this center in Naples during this pandemic period immediately showed us the complexity of the approach to these diseases during COVID-19. In the first 6 months after opening, we had 91 admissions (15.7 per month). Most patients were found to have ALS

Francesco Barbato

francesco.barbato@centrocliniconemo.it

1 NeuroMuscular Omnicentre (NEMO), Serena Onlus, A.O.R.N. dei Colli, "V. Monaldi", Naples, Italy

2 Department of Advanced Medical and Surgical Sciences, University of Campania “Luigi Vanvitelli”, Naples, Italy

3 Department of Woman, Child and General and Specialized Surgery, University of Campania "Luigi Vanvitelli", Naples, Italy and myotonic dystrophy (46 and 14 patients, respectively $51 \%$ and $14 \%$ of all). The main reason for admission was acute respiratory failure ( 23 patients, $26 \%$ of all) with the necessity to immediate noninvasive ventilation, emergency bronchoscopy or unfortunately sometimes intubation and transferring to intensive care unit (ICU) (17 patients, 18\% of all). Other causes were found to be pneumonia with fever (17 patients, $18 \%$ of all) and a severe state of clinical malnutrition (12 patients, $13 \%$ of all) started at home for a progressive dysphagia or for inability to stop non-invasive ventilation. In particular for about 1 month, two patients preferred to use continuously non-invasive ventilation for $24 \mathrm{~h}$ a day rather than appropriately feed themselves, for fear of the virus SARS COVID2. All patients with fever and pneumonia delayed access to treatment for at least 5 days due to the COVID-19 pandemic. In the group of people with pneumonia and respiratory failure, it was necessary to carry out tracheostomy for seven patients. Due to the COVID-19 pandemic, these seven patients did not receive adequate clinical information on the evolution of their disease. This is due to a growing stigma that going to the hospital to carry out regular clinical follow-up poses a risk for themselves and family members to be able to contract COVID-19. On the other hand, reporting that they have fever and cough can result in isolation at home. Not to be undervalued, it is also the anguish felt by family members at the idea of going to the hospital, which they themselves oppose this choice [3]. Although, thanks to the COVID-19 emergency, it has become evident the need for a push in the strengthening of acute hospital wards, and it is not the only reality in which healthcare personnel clash every day [4]. These numbers, impressive for a private clinical center recently time opened, in a context of a pandemic, are the result of the "real world" and how the pandemic situation requires a rearrangement of not only acute care but also an enhancement in management of patients suffering from chronic diseases, to minimize access for emergencies. 
Supplementary Information The online version contains supplementary material available at https://doi.org/10.1007/s10072-022-05889-0.

\section{References}

1. De Marchi F, Cantello R, Ambrosini S et al (2020) Telemedicine and technological devices for amyotrophic lateral sclerosis in the era of COVID-19. Neurol Sci 41:1365-1367. https://doi.org/10. 1007/s10072-020-04457-8
2. Rosellini I, Vianello M, Ghazaryan A et al (2021) Virtual visits for chronic neurologic disorders during COVID-19 pandemic. Neurol Sci. https://doi.org/10.1007/s10072-021-05212-3

3. Tabary M, Abolhasani R, Araghi F et al (2021) Ethical considerations in neurology during the COVID-19 pandemic. Neurol Sci 42:437-444. https://doi.org/10.1007/s10072-020-05032-x

4. Di Lorenzo F, Ercoli T, Cuffaro L, Barbato F et al (2021) COVID19 impact on neurology training program in Italy. Neurol Sci 42:817-823. https://doi.org/10.1007/s10072-020-04991-

Publisher's note Springer Nature remains neutral with regard to jurisdictional claims in published maps and institutional affiliations. 